A - Research concept and design

B - Collection and/or assembly of data

C - Data analysis and interpretation

D - Writing the article

E - Critical revision of the article

F - Final approval of article

\section{The effect of incentive spirometry on pulmonary function recovery and satisfaction with physical therapy of cardiac surgery patients}

\author{
Volodymyr Vitomskyi*1,A-F (D), Khaled Al-Hawamdeh ${ }^{1, A-B, D}$ (iD, \\ Maryna Vitomska ${ }^{1, B, D-E}\left(\mathbb{D}\right.$, Olena Lazarieva ${ }^{1, A, F}$ (D), Olena Haidai ${ }^{2, C-D}$ \\ ${ }^{1}$ National University of Ukraine on Physical Education and Sport, Ukraine \\ ${ }^{2}$ Bogomolets National Medical University, Ukraine
}

*Correspondence: Volodymyr Vitomskyi; National University of Ukraine on Physical Education and Sport, Ukraine; email: vitomskiyvova@gmail.com

\title{
Abstract
}

Introduction: The assessment of patient satisfaction with the received physical therapy (PT) is a necessary part of PT system analysis. The purpose of the study was to investigate the effect of incentive spirometry on pulmonary function recovery and the level of satisfaction with PT in patients undergoing cardiac surgery.

Materials and methods: 70 patients of both sexes were randomly divided into control group (CG) and training group (TG). Both groups received standardized PT (early mobilization, therapeutic exercises, coughing). TG patients also used Tri-Ball breathing exerciser (flow-oriented incentive spirometry). Outcome Measures: the indicators of pulmonary function and satisfaction with PT results on the 7th postoperative day.

Results: Pulmonary function results were statistically the same in CG and TG both before the surgery and on the seventh postoperative day. According to the results of the assessment of patient satisfaction with PT, none of the groups showed advantages in any of the seventeen items. Both groups of patients showed high scores in all items of PT satisfaction questionnaire. The overall score had no statistical difference in the groups as well: $82.5(79 ; 85)$ points in CG and $80.5(74.75 ; 85)$ points in TG $(\mathrm{p}=0.315)$ out of a maximum of 85 points.

Conclusions: The investigation of pulmonary function and patient satisfaction with the received physical therapy did not confirm the effect of flow-oriented incentive spirometry included in the physical therapy program on the level of satisfaction and pulmonary function test results.

\section{Keywords: physical therapy, pulmonary rehabilitation, respiratory system, therapeutic exercises}

\section{Introduction}

Physical therapy of cardiac and cardiac surgery patients remains an important healthcare sphere owing to the prevalence of cardiac pathology, its complications and comorbidity [1-3]. Decreased oxygenation [4], pulmonary function [5], respiratory muscle strength
$[4,6,7]$, as well as radiological changes such as atelectasis $[8,9]$ are typical postoperative changes among cardiac surgery (CS) patients. Therefore, physical therapy is routinely offered to the patients following CS. PT of cardiac surgery patients includes early mobilization, thoracic, upper and lower extremities range of motion exercises, chest physiotherapy (breathing exercises, 
chest wall vibrations, percussions, coughing/huffing techniques, positioning, lying on side, postural drainage) and relaxation techniques [10-13].

Most surveys focused on PT of cardiac surgery patients study the ways to improve restoration of their pulmonary function [4,14], functional capacity $[4,15]$, respiratory muscle strength $[4,16]$, dynamic of intensive care unit (ICU) and hospital length of stay $[7,14]$, as well as elimination of postoperative pulmonary complications $[1,8,9]$ with the help of additional interventions within standardized PT program.

However, the assessment of patient satisfaction (PS) with the received PT is currently a necessary part of physical therapy system analysis at any institution. The level of PS has become an important indicator of service quality in patient-centred health care systems [17-20]. The growing interest in measuring PS is in line with market relations in the sphere of health care, and competition $[21,22]$, which are the factors promoting service quality improvement. Besides, satisfied patients are more likely to seek for additional services and recommend the hospital to their friends [22].

Therefore, the assessment of satisfaction of cardiac surgery patients with the received PT is a relevant subject of research. Besides, studying the effect of additional PT interventions within standardized programs on satisfaction indicators is necessary because it can happen that PS will improve, with pulmonary function or functional capacity, for example, having no statistical changes due to additional intervention.

For instance, deep breathing exercise, incentive spirometry [23-26], inspiratory resistance-positive expiratory pressure [27] were reported to have no impact on pulmonary function recovery and frequency of complications. However, the investigation of physical therapists practical activity confirms high level of implemented approaches in pulmonary PT, which have an unconfirmed or refuted beneficial effect in PT of cardiac surgery patients [12]. Therefore, the study of other criteria that may be improved with the help of additional respiratory PT is relevant and may help to substantiate the fact that physical therapists use respiratory PT with unconfirmed efficacy. In particular, such criteria include the level of satisfaction with PT, anxiety or indicators of therapeutic alliance formation.

Surveys of physical therapists found that incentive spirometry is widely used in practice $[12,28,29]$. One of the studies stated that this is caused by the fact that patients like to exercise with the breathing exerciser [30]. Therefore, we decided to analyze two aspects: 1 - pulmonary function test to supplement and confirm the consistency of obtained results with the results of the previous research; 2 - the role of additional respiratory $\mathrm{PT}$ in patient satisfaction.
The purpose of the study was to investigate the effect of incentive spirometry on pulmonary function recovery and the level of satisfaction with PT in patients undergoing cardiac surgery.

\section{Materials and methods}

The study included 70 patients of both sexes, who were submitted to undergoing CS. All procedures were performed by median sternotomy on cardiopulmonary bypass with cardioplegic arrest. The exclusion criteria were: patients with unstable angina pectoris at the moment of selection or during the program, congestive decompensated heart failure, lack of intellectual capacity, complex ventricular and uncontrolled arrhythmia, uncontrolled high blood pressure, cerebrovascular accident, artificial lung ventilation less than 24-hour.

The patients were randomly divided (at 1:1 ratio; envelope method) into control group (CG) and training group (TG). The study protocol was approved by the institutional review board and the local medical ethics committee (Ethics Committee approval - protocol №1 of 21.01.2020). All patients were informed about the study protocol and gave written informed consent.

Both groups received standardized PT (early mobilization; therapeutic exercises; coughing). Before the surgery, the patients were briefly consulted by a physical therapist on the aims and content of PT and activation algorithm after the surgery. The postoperative protocol of PT called for the following practice of patient's early mobilization: sitting on the bed with the legs dangling on the 1 postoperative day (POD); standing (getting up with the help and under the control of a physical therapist, holding on a medical movable walker; agreed with an anesthesiologist) and on-the-spot walking if feasible on the 1-2 POD; on-the-spot walking, walking within the ward on the 2 POD; walking in the hospital corridor on the 3 POD; walking up and down the stairs on the 4-5 PODs. Besides, all patients performed therapeutic exercises with a physical therapist and therapeutic walking under the control of a physical therapist. Sessions (about 20 minutes each) with a physical therapist were conducted 2 times a day on the 1 and 2 PODs, 1-2 times on the 3 POD, 1 time starting from the 4 POD. In case of a necessity (patient's condition, the need for motivation), physical therapist could increase the number and the length of the sessions. PT was conducted by two physical therapists in equal amount in both groups. Each physical therapist was assigned to each group in turn.

The groups differed in respiratory therapy: TG patients additionally performed respiratory exercises with a Tri-Ball breathing exerciser (at least three repetitions 
of 10 strong, full and rapid inhalations through the breathing exerciser at the sessions with a physical therapist, as well as received recommendations to perform 3 sets with 10 repetitions every hour to lift all three balls of the breathing exerciser $(600,900$ and $1200 \mathrm{cc} / \mathrm{sec})$ with each inhalation. All participants in TG recorded their daily inspiratory muscle workout in the diaries. If a participant was not able to lift all three balls this was not considered a treatment failure, since selecting participants on this basis would contribute to the concentration of patients with better scores in TG. At the same time, such selection would be impossible in CG.

TG patients were asked to start from a lower lung volume before starting the forceful inahaltion. Training with breathing exerciser started from the 1 POD. During the 1 and 2 PODs the exercises performed every hour were supervised by medical staff, afterwards the patient recorded independently performed exercises in the diary.

Demographic variables, clinical history, the results of pulmonary function test were recorded on entry to the trial. Both groups were submitted to pulmonary function test (PFT) before the surgery and on the 7 POD. The patients performed at least 3 PFT attempts using Spirodoc MIR spirograph and Winspiro PRO software. Individual rates were calculated automatically according to Knudson/ European Respiratory Society. The personal performimg the spirometry was blinded regarding participants group allocation.

Satisfaction was assessed with the help of an adapted version of the survey questionnaire, developed and validated by Monnin and Perneger [31] and endorsed by the American Physical Therapy Association. The adapted version of the questionnaire was presented in other studies [32] and corresponded to the conditions (inpatients PT) and objectives of the current study. The questionnaire consisted of 17 questions with 10 questions pertaining to the treatment process (including interpersonal factors), 2 questions pertaining to logistics, 2 questions pertaining to organizational factors, and the remaining 3 were general questions [32]. Patients filled in the questionnaire independently on the 7 POD without seeking for the assistance of consultants, which was available if needed. The answers were distributed on a Likert scale from 1 to 5 points: "strongly agree" - 5 points, "agree" -4 points, "uncertain" -3 points, "disagree" - 2 points, "strongly disagree" - 1 point.

\section{Statistical analysis}

The materials of the research were processed in IBM SPSS 21 program of statistical analysis. Mathematical processing of numerical data was fulfilled with the help of variation statistics. The analysis of quantitative indicators distribution's correspondence to the law of normal distribution was checked by Shapiro-Wilk test (W). Mean value and root-mean-square deviation $(\bar{x} \pm \mathrm{S})$ were calculated for the results of indicators that corresponded to the law of normal distribution. Median value (Me) and upper and lower quartiles $(25 \% ; 75 \%)$ were calculated for the indicators with a non-normal distribution. Student's t-test (for independent groups) was used to measure the significance of the difference, provided there was a normal distribution of study results; Mann-Whitney U test (for independent groups) and $\chi^{2}$ criterion were used provided the indicators had a distribution other than normal.

Power analysis and measuring sample size (n) were conducted according to forced vital capacity indicator with the following values: $\alpha=0.05, \beta=0.1$, clinically significant improvement - 12\% (similar to clinically significant improvement of forced vital capacity of the lungs with bronchodilator response [33]), standard deviation $-12 \%$ [34] and $15 \%$. Consequently, the calculated n comprised 21 and 32.8, which is consistent with the studied samples.

\section{Results}

CG included 27 males and 7 females, and TG included 21 males and 15 females $(p=0.058)$. Significant differences in anthropometric data, NYHA functional class, time indicators of the operation were not found (Tab. 1). Besides, the results of the pulmonary function test had no statistical difference in the groups both before the operation and on the seventh POD. None of the patients had significant pulmonary complications aggravating the process of postoperative recovery. Thus, the level of PS with the received PT may be the criterion that will justify the efficacy of additional pulmonary PT performed with the help of Tri-Ball breathing exerciser.

Comparison of the questionnaire results did not confirm statistical advantages of any of the groups in any questionnaire item (Tab. 2). All questionnaire items had high scores, as the vast majority or all the patients chose "strongly agree" or "agree" answers, which corresponded to the maximum scores. "Uncertain" answer was chosen very rarely (usually by one respondent out of all) and only in seven items.

The total score had no statistical difference in the groups as well: $82.5(79 ; 85)$ points in $\mathrm{CG}$ and 80.5 (74.75; 85) points in TG $(\mathrm{p}=0.315)$.

Among the considered questionnaire items, the highest scores were in the first (explaining the reason for PT), seventh (friendliness and courtesy of physical therapists) and thirteenth (respect from physical therapists) items, whereas the lowest scores were in the fifth 
Tab. 1. The main characteristics of the samples

\begin{tabular}{|c|c|c|c|c|}
\hline \multicolumn{2}{|l|}{ Indicators } & $\mathrm{CG}(\mathrm{n}=34)$ & $\mathrm{TG}(\mathrm{n}=36)$ & $\mathrm{p}$ \\
\hline \multicolumn{2}{|l|}{ Age (years) } & $62.5(53 ; 68.5)$ & $64(55.3 ; 70)$ & $0.427^{*}$ \\
\hline \multicolumn{2}{|l|}{ Body weight (kg) } & $83.00 \pm 13.85$ & $81.74 \pm 15.71$ & $0.723^{\#}$ \\
\hline \multicolumn{2}{|l|}{ Body length (cm) } & $170.47 \pm 9.24$ & $167.28 \pm 9.53$ & $0.160^{\#}$ \\
\hline \multicolumn{2}{|c|}{ Body mass index $\left(\mathrm{kg} / \mathrm{m}^{2}\right)$} & $28.51 \pm 3.94$ & $29.22 \pm 5.10$ & $0.520^{\#}$ \\
\hline \multicolumn{2}{|l|}{$\mathrm{EF}(\%)$} & $55(47.8 ; 58.5)$ & $54.5(47 ; 60)$ & $0.958^{*}$ \\
\hline \multicolumn{2}{|l|}{ AH (degree) } & $2(0 ; 3)$ & $2(2 ; 3)$ & $0.234^{*}$ \\
\hline \multicolumn{2}{|l|}{ NYHA (class) } & $3(2 ; 3)$ & $3(2 ; 3)$ & $0.680^{*}$ \\
\hline \multicolumn{2}{|c|}{ AVL duration (hour) } & $7(6 ; 9,5)$ & $8(6 ; 11)$ & $0.181^{*}$ \\
\hline \multicolumn{2}{|c|}{ Operation duration (min) } & $368(327 ; 420)$ & $337(285 ; 428)$ & $0.312^{*}$ \\
\hline \multicolumn{2}{|c|}{ CPB duration (min) } & $171.85 \pm 55.89$ & $186.31 \pm 57.37$ & $0.290^{\#}$ \\
\hline \multirow{5}{*}{ Before operation } & $\mathrm{VC}, \%$ predicted & $102.32 \pm 15.98$ & $105.33 \pm 14.05$ & $0.405^{\#}$ \\
\hline & FVC, $\%$ predicted & $100.71 \pm 14.94$ & $102.14 \pm 14.93$ & $0.689^{\#}$ \\
\hline & $\mathrm{FEV}_{1} / \mathrm{VC}, \%$ & $77.22 \pm 9.65$ & $76.43 \pm 8.11$ & $0.709^{\#}$ \\
\hline & PEF, \% predicted & $96.24 \pm 13.90$ & $98.61 \pm 18.83$ & $0.552^{\#}$ \\
\hline & PIF, \% predicted & $69.06 \pm 18.93$ & $69.64 \pm 21.85$ & $0.906^{\#}$ \\
\hline \multirow{5}{*}{ On the 7 POD } & $\mathrm{VC}, \%$ predicted & $75.56 \pm 15.25$ & $75.89 \pm 16.27$ & $0.931^{\#}$ \\
\hline & FVC, $\%$ predicted & $74.35 \pm 15.38$ & $73.92 \pm 17.51$ & $0.912^{\#}$ \\
\hline & $\mathrm{FEV}_{1} / \mathrm{VC}, \%$ & $79.35 \pm 8.39$ & $79.35 \pm 8.65$ & $1.000^{\#}$ \\
\hline & PEF, \% predicted & $78.41 \pm 18.24$ & $81.42 \pm 20.43$ & $0.519^{\#}$ \\
\hline & PIF, \% predicted & $57.00 \pm 14.80$ & $57.69 \pm 18.20$ & $0.862^{\#}$ \\
\hline
\end{tabular}

Note: EF - ejection fraction; AH - arterial hypertension; AVL - artificial lung ventilation; CPB - cardiopulmonary bypass; VC - vital capacity; FVC - forced vital capacity; FEV1/ VC - Tiffeneau index; PEF - peak expiratory flow; PIF - peak inspiratory flow; POD - postoperative day; ${ }^{\#}$ - Student's t-test; ${ }^{*}$ - Mann-Whitney U-test.

(access to the necessary physical therapists regarding feedback on patients' PT procedures), second (availability of materials and equipment needed to complete the care) and third (making patients feel their diagnosis was correct) items. However, even the items with the lowest scores had the result very close to the maximum.

\section{Discussion}

Both groups of patients showed high scores among all items of PT satisfaction questionnaire. Most patients "strongly agreed" or "agreed" with the questionnaire statements, whereas just a few of them chose "uncertain" answer and no one chose "disagree" or "strongly disagree" options.

Statistical analysis did not confirm the effect of the additional use of a breathing exerciser within PT program on PS. Thus, PS is probably based on the quality of physical therapist's work, his/her communication skills and the ability to connect to patients.
Taking into account that the maximum theoretical satisfaction score is 85 points and the minimum score is 17 points (with "strongly disagree" answers in all items), it must be noted that mean value of the total score comprised $94.7 \%$ out of the maximum score in $\mathrm{CG}$, and $91.2 \%$ in $\mathrm{TG}$.

According to our data, this study is one of the few that properly assessed satisfaction with PT among inpatient cardiac surgery patients, and the only one that analyzed the effect of additional flow-oriented incentive spirometry within the standardized inpatient PT protocol on PS. At the same time, according to one of the studies, preoperative inspiratory muscle training were highly scored by the patients on 10-point satisfaction and motivation scales $(8.1(0.6)$ and $8.4(0.9)$, respectively) [8].

The comparison of the effect of a high and low frequency exercise therapy program on the level of satisfaction with PT of the patients after CABG was presented in the study of van der Peijl et al. [35]. The researchers confirmed the advantages of high frequency 
Tab. 2. Assessment results of satisfaction with PT

\begin{tabular}{|c|c|c|c|c|c|c|}
\hline \multirow{2}{*}{ Items } & & \multirow{2}{*}{ Groups } & \multicolumn{3}{|c|}{ Answers, \% } & \multirow{2}{*}{$\mathrm{p}\left(\chi^{2}\right.$ criterion $)$} \\
\hline & & & Strongly agree & Agree & Uncertain & \\
\hline \multirow{2}{*}{1} & \multirow{2}{*}{$\begin{array}{l}\text { Physiotherapists were good about explaining } \\
\text { the reason for my physiotherapy }\end{array}$} & CG & 91.2 & 8.8 & - & \multirow{2}{*}{0.204} \\
\hline & & TG & 80.6 & 19.4 & - & \\
\hline \multirow{2}{*}{2} & \multirow{2}{*}{$\begin{array}{l}\text { I think the physiotherapist had materials and } \\
\text { equipment needed to complete my care }\end{array}$} & CG & 64.7 & 35.3 & - & \multirow{2}{*}{0.518} \\
\hline & & TG & 69.4 & 27.8 & 2.8 & \\
\hline \multirow{2}{*}{3} & \multirow{2}{*}{$\begin{array}{l}\text { Physiotherapists always made me feel their } \\
\text { diagnosis was correct }\end{array}$} & CG & 64.7 & 35.3 & - & \multirow{2}{*}{0.178} \\
\hline & & TG & 47.2 & 47.2 & 5.6 & \\
\hline \multirow{2}{*}{4} & \multirow{2}{*}{$\begin{array}{l}\text { The physiotherapists were thorough in } \\
\text { treating and examining me }\end{array}$} & CG & 88.2 & 11.8 & - & \multirow{2}{*}{0.155} \\
\hline & & TG & 75 & 25 & - & \\
\hline \multirow[b]{2}{*}{5} & \multirow{2}{*}{$\begin{array}{l}\text { I had easy access to the physiotherapists } \\
\text { I needed regarding feedback on my } \\
\text { physiotherapy procedures }\end{array}$} & CG & 52.9 & 47.1 & - & \multirow[b]{2}{*}{0.520} \\
\hline & & TG & 58.3 & 38.9 & 2.8 & \\
\hline \multirow[b]{2}{*}{6} & \multirow{2}{*}{$\begin{array}{l}\text { I didn't have to wait for a long period before } \\
\text { being attended to by the physiotherapists } \\
\text { after I was referred to them }\end{array}$} & CG & 76.5 & 23.5 & & \multirow[b]{2}{*}{0.167} \\
\hline & & TG & 61.1 & 38.9 & - & \\
\hline \multirow{2}{*}{7} & \multirow{2}{*}{$\begin{array}{l}\text { My physiotherapists treated me in a very } \\
\text { friendly and courteous manner }\end{array}$} & $\mathrm{CG}$ & 94.1 & 5.9 & - & \multirow{2}{*}{0.090} \\
\hline & & $\mathrm{TG}$ & 80.6 & 19.4 & - & \\
\hline \multirow{2}{*}{8} & \multirow{2}{*}{$\begin{array}{l}\text { Those who provided my physiotherapy care } \\
\text { always took their time when they treated me }\end{array}$} & CG & 73.5 & 26.5 & - & \multirow{2}{*}{0.181} \\
\hline & & TG & 58.3 & 41.7 & - & \\
\hline 0 & The physiotherapists always acknowledged & CG & 88.2 & 11.8 & - & 0137 \\
\hline$y$ & what I told them & TG & 69.4 & 27.8 & 2.8 & 0.151 \\
\hline 10 & I had no doubts about the ability of the & CG & 73.5 & 26.5 & - & 03 \\
\hline 10 & physiotherapists who treated me & TG & 63.9 & 36.1 & - & 0.385 \\
\hline & I felt confident that I was receiving the & CG & 85.3 & 14.7 & - & \\
\hline 11 & $\begin{array}{l}\text { physiotherapy I need without being setback } \\
\text { financially }\end{array}$ & TG & 63.9 & 33.3 & 2.8 & 0.104 \\
\hline 12 & I was very satisfied with the physiotherapy & CG & 79.4 & 20.6 & & 0196 \\
\hline & care I received & TG & 61.1 & 36.1 & 2.8 & 0.190 \\
\hline 12 & The physiotherapists who treated me gave me & CG & 91.2 & 8.8 & - & (2) \\
\hline 13 & respect & TG & 77.8 & 22.2 & - & 0.124 \\
\hline 14 & During my physiotherapy I was allowed to & CG & 88.2 & 11.8 & - & 0094 \\
\hline & say everything that I thought was important & TG & 72.2 & 27.8 & - & \\
\hline 15 & The physiotherapists who treated me had & CG & 79.4 & 20.6 & - & 0058 \\
\hline $1 J$ & a genuine interest in me as a person & TG & 58.3 & 41.7 & - & 0.050 \\
\hline 16 & I was fully compliant with the physiotherapy & CG & 70.6 & 26.5 & 2.9 & ? \\
\hline 10 & treatment I received & TG & 58.3 & 41.7 & - & 0.200 \\
\hline & Due to my level of satisfaction my & CG & 79.4 & 20.6 & - & \\
\hline 17 & $\begin{array}{l}\text { compliance to the physiotherapy came } \\
\text { naturally }\end{array}$ & TG & 66.7 & 33.3 & - & 0.231 \\
\hline
\end{tabular}


group patients in treatment variables (for 4 of 6 questions), information (for 3 of 4 questions), empathy ( $p<0.05$ for 2 of 6 questions) and the total score of the program ( 8.3 vs. 7.6 points on a 10-point scale). At the same time, $79 \%$ of patients from the low frequency group wanted to receive PT on weekends, and 61\% from the high frequency group, on the contrary, wanted to have less PT on the weekend. Besides, there are attempts to assess the experience of patients in PT in the sphere of cardiac surgery.

For example, one of the studies reported that only $16.7 \%$ had contact with the physiotherapist before surgery; only $2.9 \%$ of patients reported having received educational guidelines about the postoperative period. However, $56.8 \%$ rated the PT as good and $100 \%$ of patients reported believing that physiotherapy could improve their health status [36]. One of the studies confirmed better satisfaction with medical services in patients who received PT after CABG [37]. Some studies confirmed beneficial effect of massage therapy on satisfaction of cardiac surgery patients, as well as on pain, anxiety, muscular tension and relaxation, measured on visual analog scales $[38,39]$. On the other hand, our study supplemented the available data on the quality of PT and the level of PS. In particular, preceding studies investigated this aspect in patients with musculoskeletal disorders $[40,41]$.

In general, the studies confirm high satisfaction of patients having musculoskeletal disorders with outpatient PT programs in Europe and North America [40,41]. At the same time, the authors of a systematic review noted that interpersonal attributes of the therapist and the process of PT are key determinants of PS. An unexpected finding was that treatment outcome was infrequently and inconsistently associated with PS. Physical therapists can enhance the quality of patient-centered care by understanding and optimizing these determinants of PS [41]. This statement is partially consistent with our results, though investigation of the effect of pulmonary function recovery level on PT satisfaction requires additional analysis.

Besides, one of the factors affecting the level of PS are age $[42,43]$ and specificities of the attitude to the disease [40]. It was found that older clients tended to rate accessibility of PT services more positively and were more likely to agree that their physical therapist had helped them to understand and manage their health condition better. The findings suggest that age group may be correlated with satisfaction with access to services, but is not related to satisfaction with health-related outcomes [42]. Furthermore, there is a significant impact of tangibles, reliability, responsiveness, assurance and empathy on satisfaction of PT [43].

\section{Study limitations}

The weakness of the study is that the questions included in the questionnaire are mainly focused on the physical therapist. Therefore, to assess more distinct and specific impact of additional respiratory physical therapy, attention should be paid to questionnaire items № 2 (availability of the necessary equipment), № 4 (thoroughness of treatment) and № 10 (ability of the physiotherapists, since lack of respiratory exercises could influence patient's assessment). To assess the impact on overall satisfaction, attention should be paid to items №12, № 17 and the overall score.

\section{Conclusion}

Indicators of PFT and PS with physical therapy were statistically the same in both groups. All satisfaction questionnaire items had high scores. The total score was very close to the maximum level and had no statistical difference in the groups. Thus, the study did not confirm the effect of flow-oriented incentive spirometry included in the physical therapy program on the level of satisfaction.

\section{Funding}

This research received no external funding.

\section{Conflicts of interest}

The authors declare no conflict of interest.

\section{References}

1. Vitomskyi V. The impact of mobilization and other factors on pleural effusion in patients undergoing cardiac surgical procedures. J Phys Educ Sport. 2020; 20 (Suppl 3): 2167-73.

2. Sarna I, Smolis-Bąk E. Comparison of cardiac rehabilitation programmes including patients' education in selected institutions in Portugal and Poland. Adv Rehab. 2018; 32(2): 23-32.

3. Bielecka-Kowal K, Jóźwik S, Woźniewski M. Interval training effects in patients with implantable cardioverter defibrillator depending on their exercise tolerance level. Adv Rehab. 2020; 34(1): 19-24.

4. Ferreira PE, Rodrigues AJ, Evora PR. Effects of an inspiratory muscle rehabilitation program in the postoperative period of cardiac surgery. Arq Bras Cardiol. 2009; 92(4): 275-82.

5. Johnson D, Hurst T, Thomson D, et al. Respiratory function after cardiac surgery. J Cardiothorac Vasc Anesth. 1996; 10(5): 571-7. 
6. Weiner P, Zeidan F, Zamir D, et al. Prophylactic inspiratory muscle training in patients undergoing coronary artery bypass graft. World J Surg. 1998; 22(5): 427-31.

7. Mans CM, Reeve JC, Elkins MR. Postoperative outcomes following preoperative inspiratory muscle training in patients undergoing cardiothoracic or upper abdominal surgery: a systematic review and meta analysis. Clin Rehabil. 2015; 29(5): 426-38.

8. Hulzebos EH, Helders PJ, Favié NJ, De Bie RA, de la Riviere AB, Van Meeteren NL. Preoperative intensive inspiratory muscle training to prevent postoperative pulmonary complications in high-risk patients undergoing CABG surgery: a randomized clinical trial. JAMA. 2006; 296(15): 1851-7.

9. Cordeiro ALL, Melo TAD, Neves D, et al. Inspiratory muscle training and functional capacity in patients undergoing cardiac surgery. Braz J Cardiovasc Surg. 2016; 31(2): 140-4.

10. Stein R, Maia CP, Silveira AD, Chiappa GR, Myers J, Ribeiro JP. Inspiratory muscle strength as a determinant of functional capacity early after coronary artery bypass graft surgery. Arch Phys Med Rehabil. 2009; 90(10): 1685-91.

11. Westerdahl E, Moller M. Physiotherapy-supervised mobilization and exercise following cardiac surgery: A national questionnaire survey in Sweden. J Cardiothorac Surg. 2010; 25(5): 67.

12. Lomi C, Westerdahl E. Physical therapy treatment after cardiac surgery: a national survey of practice in Greece. J Clin Exp Cardiolog. 2013; S7(004): 1-5.

13. Vitomskiy V, Hruzevych I, Salnykova S, et al. The physical development of children who have a functionally single heart ventricle as a basis for working physical rehabilitation technology after a hemodynamic correction. J Phys Educ Sport. 2018; 18(2): 614-7.

14. Savci S, Degirmenci B, Saglam M, et al. Short-term effects of inspiratory muscle training in coronary artery bypass graft surgery: a randomized controlled trial. Scand Cardiovasc J. 2011; 45(5): 286-93.

15. Cargnin C, Karsten M, da Costa Guaragna JCV, Dal Lago P. Inspiratory muscle training after heart valve replacement surgery improves inspiratory muscle strength, lung function, and functional capacity: a randomized controlled trial. J Cardiopulm Rehabil Prev. 2019; 39(5): E1-E7.

16. Kodric M, Trevisan R, Torregiani C, et al. Inspiratory muscle training for diaphragm dysfunction after cardiac surgery. J Thorac Cardiovasc Surg. 2013; 145(3): 819-23.

17. Hush JM, Cameron K, Mackey M. Patient satisfaction with musculoskeletal physical therapy care: a systematic review. Phys Ther. 2011; 91(1): 25-36.
18. Brown JB, Stewart MA, McCracken E, et al. The patient-centred clinical method. 2. Definition and application. Fam Pract. 1986; 3: 75-9.

19. Law M, Baptiste S, Mills J. Client-centred practice: What does it mean and does it make a difference? Can J Occup Ther. 1995; 62: 250-7.

20. Donabedian A. The quality of care: How can it be assessed? JAMA. 1988; 260: 1743-8.

21. Bruce B, Fries JF. The Stanford Health Assessment Questionnaire: dimensions and practical applications. Health Qual Life Outcomes. 2003; 9(1): 20.

22. Beattie PF, Pinto MB, Nelson MK, et al. Patient satisfaction with outpatient physical therapy: instrument validation. Phys Ther. 2002; 82: 557-65.

23. Jenkins SC, Soutar SA, Loukota JM, Johnson LC, Moxham J. Physiotherapy after Coronary artery surgery: are breathing exercises necessary? Thorax. 1989; 44(8): 634-9.

24. Jenkins SC, Soutar SA, Loukota JM, Johnson LC, Moxham J. A comparison of breathing exercises, incentive spirometry and mobilisation after coronary artery surgery. Physiother Theory Pract. 1990; 6: 117-26.

25. Dull JL, Dull WL. Are maximal inspiratory breathing exercises or incentive spirometry better than early mobilisation after cardiopulmonary bypass? Phys Ther. 1983; 63: 655-9.

26. Renault JA, Costa-Val R, Rosseti MB, Houri Neto M. Comparison between deep breathing exercises and incentive spirometry after CABG surgery. Rev Bras Cir Cardiovasc. 2009; 24: 165-72.

27. Westerdahl E, Lindmark B, Almgren SO, Tenling A. Chest physiotherapy after coronary artery bypass graft surgery - a comparison of three different deep breathing techniques. J Rehabil Med. 2001; 33(2): 79-84.

28. Jenkins SC, Soutar AS. A survey into the use of incentive spirometry following coronary artery by-pass graft surgery. Physiotherapy. 1986; 72: 492-3.

29. Overend TJ, Anderson CM, Jackson J, Lucy SD, Prendergast M, Sinclair S. Physical therapy management for adult patients undergoing cardiac surgery: a Canadian practice survey. Physiother Can. 2010; 62(3): 215-21.

30. Reeve J, Ewan S. The physiotherapy management of the coronary artery bypass graft patient: a survey of current practice throughout the United Kingdom. Assoc Chart Physiother Resp Care. 2006; 27: 35-45.

31. Monnin D, Perneger TV. Scale to measure patient satisfaction with physical therapy. Phys Ther. 2002; 82(7): 682-91.

32. Ampiah PK, Ahenkorah J, Karikari M. Patients' satisfaction with inpatient orthopedic physiotherapy services at a Tertiary Hospital in Ghana. J Patient Exp. 2019; 6(3): 238-46. 
33. Quanjer PH, Tammeling GJ, Cotes JE, Pedersen OF, Peslin R, Yernault JC. Lung volumes and forced ventilatory flows. Report Working Party "Standardization ot Lung Function Tests". European Coal and Steel Community. Eur Respir J. 1993; 6(Suppl.16): 5-40.

34. Westerdahl E, Lindmark B, Eriksson T, Hedenstierna G, Tenling A. Deep-breathing exercises reduce atelectasis and improve pulmonary function after coronary artery bypass surgery. CHEST. 2005; 128(5): 3482-8.

35. Van der Peijl ID, Vliet Vlieland TP, Versteegh MI, Lok JJ, Munneke M, Dion RA. Exercise therapy after coronary artery bypass graft surgery: a randomized comparison of a high and low frequency exercise therapy program. Ann Thorac Surg. 2004; 77(5): 1535-41.

36. Lima P, Cavalcante H, Rocha Â, Brito R. Physical therapy in postoperative cardiac surgery: the patient's perception. Braz J Cardiovasc Surg. 2011; 26(2): 244-9.

37. Brown D. Is participation in cardiac rehabilitation programs associated with better quality of life and return to work after coronary artery bypass operations?. The Israeli CABG Study. Isr Med Assoc J. 2001; 3: 399-403.
38. Braun LA, Stanguts C, Casanelia L, et al. Massage therapy for cardiac surgery patients - a randomized trial. J Thorac Cardiovasc Surg. 2012; 144(6): 1453-9.

39. Bauer BA, Cutshall SM, Wentworth LJ, et al. Effect of massage therapy on pain, anxiety, and tension after cardiac surgery: a randomized study. Complement Ther Clin Pract. 2010; 16(2): 70-5.

40. Fedorenko S, Vitomskyi V, Lazarieva O, et al. Influence specificities of the type of attitude towards a disease on physical therapy satisfaction among the orthopedic profile patients and the possibilities of attitude improvement. J Physic Educ Sport. 2020; 20(2): 896-904.

41. Hush JM, Cameron K, Mackey M. Patient satisfaction with musculoskeletal physical therapy care: a systematic review. Phys Ther. 2011; 91(1): 25-36.

42. McKinnon AL. Client satisfaction with physical therapy services: does age make a difference? Phys Occup Ther Geriatr. 2001; 19(2): 23-37.

43. Al Azmi N, Al-Lozi M, Al-Zu'bi Z, Dahiyat S, Masa'deh R. Patients attitudes toward service quality and its impact on their satisfaction in physical therapy in KSA hospitals. Eur J Soc Sci. 2012; 34(2): 300-14. 